TAIWANESE JOURNAL OF MATHEMATICS

Vol. 14, No. 6, pp. 2329-2342, December 2010

This paper is available online at http://www.tjm.nsysu.edu.tw/

\title{
VISCOSITY-TYPE APPROXIMATION METHOD FOR EFFICIENT SOLUTIONS IN VECTOR OPTIMIZATION
}

\author{
Thai Doan Chuong and Jen-Chih Yao*
}

\begin{abstract}
The paper is devoted to developing the viscosity-type approximation algorithm of finding efficient solutions to the vector optimization problem for a mapping between finite dimensional Hilbert spaces with respect to the partial order induced by a pointed closed convex cone. We prove that under some suitable conditions either the sequence generated by our method converges to an efficient solution or its cluster points belong to the set of all efficient solutions of this problem.
\end{abstract}

\section{INTRODUCTION}

The purpose of this paper is to develop and justify the iterative algorithm of the so-called viscosity-type approximation to find efficient (or Pareto) solutions for a general class of vector optimization problems in finite-dimensional Hilbert spaces described as follows.

Let $X$ and $Y$ be finite dimensional Hilbert spaces. Given a pointed closed convex cone $C \subset Y$, we consider the partial order $\preceq_{C}$ in $Y$ defined by

$$
y \preceq_{C} y^{\prime} \text { if and only if } y^{\prime}-y \in C .
$$

In what follows we are not going to impose the nonempty interior assumption on the ordering cone $C$, but to compare with the previous constructions and results recall that for int $C \neq \emptyset$, i.e., if the cone $C$ is solid, the "weak" counterpart of (1.1) is defined by

$$
y \prec_{C} y^{\prime} \text { if and only if } y^{\prime}-y \in \operatorname{int} C .
$$

Received June 5, 2009.

2000 Mathematics Subject Classification: 49J52, 49M99, 65K05, 90C25.

Key words and phrases: Vector optimization, Efficient solution viscosity-type approximation method, Asymptotic function Asymptotic cone.

This work was supported by the Grant NSC 96-2628-E-110-014-MY3 and the National Program in Basic Sciences (Vietnam).

*Corresponding author. 
Consider further an extended vector-valued mapping $F: X \rightarrow Y \cup\left\{+\infty_{C}\right\}$ where the symbol $+\infty_{C}$ in the extended image space $\bar{Y}$ is defined and discussed in Section 2. The main model of our study in this paper is the vector optimization problem (VOP) formalized as

$$
\min _{C}\{F(x) \mid x \in X\},
$$

where the " $\min _{C}$ " is understood with respect to the ordering relation $\preceq_{C}$ from (1.1).

We write $\bar{x} \in \operatorname{Argmin}_{\mathrm{C}}\{F(x) \mid x \in X\}$ to indicate that $\bar{x}$ is an efficient (or Pareto) solution of (1.3) if $\bar{x} \in X$ and there is no $x \in X$ satisfying $F(x) \preceq_{C} F(\bar{x})$ with $F(x) \neq F(\bar{x})$. When int $C \neq \emptyset$, an element $\bar{x} \in X$ is weakly efficient (or weak Pareto) for this problem if there is no $x \in X$ satisfying $F(x) \prec_{C} F(\bar{x})$.

Our main concern is to analyze the methods for finding efficient solutions of problem (1.3). There are many publications devoted to the study of various methods for vector optimization problems of finding efficient or/and weakly efficient solutions (see, e.g., [5-9, 11-15] and the references therein). Let us briefly discuss some of them.

The steepest descent method for weakly efficient solutions of multiobjective optimization with $C=\mathbb{R}_{+}^{n}$ being the nonnegative orthant of $\mathbb{R}^{n}$ was dealt with in [12]; the same method for partial orders given by rather general cones in $\mathbb{R}^{n}$ was presented in [14] and for Hilbert settings was given in [10]. An extension of the projected gradient method to the case of constrained vector optimization with the order given by a general cone in $\mathbb{R}^{n}$ can be found in [13]. Recently, a geometrical interpretation of the weighting method for constrained vector optimization has been done in [15].

It is worth noticing that Bonnel et al. [5] constructed a proximal point algorithm to investigate a convex vector optimization problem (1.3) of finding weakly efficient solutions for a mapping from a Hilbert space to a Banach space. Recently, Ceng and Yao [7] studied the approximate proximal method as well as discussed an extension to Bregman function based proximal algorithms for solving a weakly efficient solution of (1.3).

The authors in [6] introduced and studied a certain hybrid approximate proximal method of finding weakly efficient solutions to the convex constrained vector optimization problem by utilizing and developing iterative processes from the fixed-point theory for nonexpansive operators and combining them with algorithms for solving some classes of monotone variational inequalities. Another results in this direction are given in [9] where the authors developed new hybrid approximate proximaltype algorithms to find efficient (or Pareto) solutions to problems of the convex constrained vector optimization in both finite-dimensional and infinite-dimensional spaces.

Very recent result in another direction is given in [8] where the authors introduced and studied the so-called generalized viscosity approximation (or viscosity- 
type approximation) method of finding weakly efficient solutions to the vector optimization problem for a mapping between finite dimensional spaces with the ordering cone $C=\mathbb{R}_{+}^{n}$ being the nonnegative orthant of $\mathbb{R}^{n}$. Under some conditions, they proved that any sequence generated by their method converges to a weakly efficient solution.

It has been well recognized that the notion of weakly efficient solutions requiring the nonempty interior assumption on ordering cones is more of some theoretical interest being rather convenient for employing conventional techniques of scalarization, separation, etc., but not being usually demanded by engineering, economic, operations research, and other important applications of multiobjective optimization. Moreover, in real-life applications it is often the case that only efficient solutions (instead of weakly efficient ones) are of interest (see, e.g., Section 2.3 in [16]).

These observations strongly motivate us to focus on efficient solutions while not weakly efficient ones to vector optimization problems. The present paper is devoted to developing the viscosity-type approximation algorithm of finding efficient solutions to vector optimization problems of (1.3). In the subsequent sections of our paper we modify the algorithm in [8] to avoid the restrictive interiority assumption in such a way that the constructed iterative sequence with the property either this sequence converges to an efficient solution or its cluster points belong to the set of all efficient solutions of the initial vector optimization problem (1.3). Among the major modifications implemented below, we mention the construction and justification of the so-called r-proper efficient solutions to the approximating problems instead of weakly efficient ones as in [8] and the previous developments.

The rest of the paper is organized as follows. Section 2 contains some preliminary material and basic definitions needed for the formulation and the proof of the main result. The last section is devoted to the study of the viscosity-type approximation algorithm. Under some suitable conditions, we prove that either the sequence generated by the method converges to some efficient solution of (1.3) or every cluster point of it is an efficient solution of this problem.

\section{PReLIMINARIES}

In this section we present the basic definitions and notations widely used in what follows and also define and discuss some constructions and standing assumptions that play important roles in establishing our main results in the subsequent sections.

Following [3], we consider the extended ordered space $\bar{Y}:=Y \cup\left\{-\infty_{C},+\infty_{C}\right\}$ and recall that a neighborhood of $+\infty_{C}$ is defined as a set $U \subset \bar{Y}$ containing $a+C \cup\left\{+\infty_{C}\right\}$ for some $a \in Y$ and its opposite $-U$ is a neighborhood of $-\infty_{C}$. The binary/ordering relations $\preceq_{C}$ and $\prec_{C}$ defined in Section 1 are extended to $\bar{Y}$ by $-\infty_{C} \preceq_{C} y \preceq_{C}+\infty_{C}$ and $-\infty_{C} \prec_{C} y \prec_{C}+\infty_{C}$ for all $y \in Y$. Observe that 
the embedding $Y \subset \bar{Y}$ is dense and continuous. The positive polar and the strict polar to $C$ are defined, respectively, by

$$
C^{+}:=\{\hbar \in Y \mid\langle y, \hbar\rangle \geq 0 \text { for all } y \in C\}
$$

and

$$
C_{s}^{+}:=\{\hbar \in Y \mid\langle y, \hbar\rangle>0 \text { for all } y \in C \backslash\{0\}\} .
$$

Given a set $\Theta \subset \bar{Y}$, define the distance function to $\Theta$ by

$$
d(y, \Theta)=\inf \{\|y-z\| \mid z \in \Theta \cap Y\}, \quad y \in Y .
$$

All the mappings $F: X \rightarrow Y \cup\left\{+\infty_{C}\right\}$ under consideration are assumed to be proper, i.e., not identically equal to $+\infty_{C}$ with the domain

$$
\operatorname{dom} F:=\left\{x \in X \mid F(x) \neq+\infty_{C}\right\} .
$$

We extend by continuity every $\hbar \in C^{+} \backslash\{0\}$ to $\bar{Y}$ by putting $\left\langle \pm \infty_{C}, \hbar\right\rangle= \pm \infty$ (see [3] for more details). A mapping $F: X \rightarrow Y \cup\left\{+\infty_{C}\right\}$ is called positively lower semicontinuous (see, [5]) if its extended real-valued scalarization $x \mapsto\langle F(x), \hbar\rangle$ is lower semicontinuous (1.s.c.) for every $\hbar \in C^{+} . F$ is called positively partially continuous if if its extended real-valued scalarization $x \mapsto\langle F(x), \hbar\rangle$ is continuous on every closed convex subset of $\operatorname{dom} F$ for every $\hbar \in C^{+} . F: X \rightarrow Y \cup\left\{+\infty_{C}\right\}$ is $C$-convex if

$F\left((1-\lambda) x+\lambda x^{\prime}\right) \preceq_{C}(1-\lambda) F(x)+\lambda F\left(x^{\prime}\right)$ for all $x, x^{\prime} \in X$ and $\lambda \in[0,1]$.

Our standing assumptions in this paper are that $C \neq\{0\}$ and that the objective mapping $F$ in (1.3) is positively lower semicontinuous, positively partially continuous and $C$-convex.

Recall that $F: \Omega \subset X \rightarrow Y$ is Gateaux differentiable at $x_{0} \in \Omega$ if there exists a matrix $D F\left(x_{0}\right)$ such that for any $v \in X$,

$$
D F\left(x_{0}\right) v=\lim _{t \rightarrow 0^{+}} \frac{F\left(x_{0}+t v\right)-F\left(x_{0}\right)}{t} .
$$

$D F\left(x_{0}\right)$ is called the Gateaux derivative of $F$ at $x_{0}$. If $F$ is Gateaux differentiable at every $x \in \Omega$, then $F$ is said to be Gâteaux differentiable on $\Omega$.

We say that $\bar{x}$ is a properly efficient solution to (1.3) and denote it by

$$
\bar{x} \in \operatorname{Argmin}_{C} p\{F(x) \mid x \in X\}
$$

if there is a pointed closed convex cone $K \subset Y$ such that $C \backslash\{0\} \subset$ int $K$ and we have $\bar{x} \in \operatorname{Argmin}_{K}\{F(x) \mid x \in X\}$. 
It follows from [5, Theorem 2.1] that

$$
\operatorname{Argmin}_{C}^{p}\{F(x) \mid x \in X\}=\bigcup_{\hbar \in C_{s}^{+}}[\operatorname{argmin}\{\langle F(x), \hbar\rangle \mid x \in X\}],
$$

provided that the objective mapping $F$ of the vector optimization problem (1.3) is $C$ convex. Moreover, we can also verify the validity of $\bar{x} \in \operatorname{Argmin}_{C}{ }^{p}\left\{F_{0}(x) \mid x \in \Omega\right\}$ if and only if $\bar{x} \in \operatorname{Argmin}_{C}{ }^{p}\left\{\widetilde{F}_{0}(x) \mid x \in X\right\}$ for the unconstrained cost mapping $\widetilde{F}_{0}: X \rightarrow Y \cup\left\{+\infty_{C}\right\}$ defined by

$$
\widetilde{F}_{0}(x)= \begin{cases}F_{0}(x) & \text { if } x \in \Omega, \\ +\infty_{C} & \text { if } x \in X \backslash \Omega,\end{cases}
$$

where $\Omega \subset X$ and $F_{0}$ is a mapping from $\Omega$ to $Y$.

Next we define, given $r \in(0,1]$, the restricted polar

$$
K_{r}:=\{\hbar \in Y \mid\langle y, \hbar\rangle \geq r\|y\| \cdot\|\hbar\| \text { for all } y \in C\}
$$

and observe that $K_{r} \backslash\{0\} \subset C_{s}^{+}$for every $r \in(0,1]$ and that $K_{r_{2}} \subset K_{r_{1}}$ whenever $0<r_{1}<r_{2} \leq 1$. We say that $\bar{x} \in X$ is a $r$-properly efficient solution to (1.3) (see [9]) if there is $\hbar \in K_{r} \backslash\{0\}$ such that $\bar{x} \in \operatorname{argmin}\{\langle F(x), \hbar\rangle \mid x \in X\}$. The set of $r$-properly efficient solutions to (1.3) is denoted by $\operatorname{Argmin}_{C}^{r p}\{F(x) \mid x \in X\}$; thus we have by definition that

$$
\operatorname{Argmin}_{\mathrm{C}}^{r p}\{F(x) \mid x \in X\}=\bigcup_{\hbar \in K_{r} \backslash\{0\}}[\operatorname{argmin}\{\langle F(x), \hbar\rangle \mid x \in X\}] .
$$

Another standing assumption imposed in this paper is as follows:

$$
\text { (H) There exists } r \in(0,1] \text { such that } K_{r} \neq\{0\} \text {. }
$$

As shown in [5, Remark 4], the property (2.6) is automatic if the interior of the polar $C^{+}$from (2.1) is nonempty. Thus it is worth mentioning to this that, as it follows from [17, Proposition 1.10], the assumption (2.6) is always fulfilled in our setting. We refer the reader to [5] where this assumption has been introduced and discussed in detail for more general settings.

Let us now recall some notions and their properties in [1]. Let $\Omega \subset X$ be a nonempty set. The asymptotic cone of $\Omega$, denoted by $\Omega_{\infty}$, is the set of all vectors $d \in X$ that are limits in the direction of the sequences $\left\{x_{n}\right\} \subset \Omega$, namely

$$
\Omega_{\infty}=\left\{d \in X \mid \exists t_{n} \rightarrow+\infty, \exists x_{n} \in \Omega \text { with } \lim _{n \rightarrow+\infty} \frac{x_{n}}{t_{n}}=d\right\} .
$$


In the case that $\Omega$ is convex and closed, for any $x_{0} \in \Omega$,

$$
\Omega_{\infty}=\left\{d \in X \mid x_{0}+t d \in \Omega \quad \forall t>0\right\} .
$$

For any proper function $f: X \rightarrow \mathbb{R} \cup\{+\infty\}$, the asymptotic function of $f$ is defined as the function $f_{\infty}$ such that epi $f_{\infty}=(\text { epi } f)_{\infty}$ where epi $f=\{(x, t) \in$ $X \times \mathbb{R} \mid f(x) \leq t\}$ is the epigraph of $f$. Consequently, we can give the analytic representation of the asymptotic function $f_{\infty}$ as follows:

$$
f_{\infty}(d)=\inf \left\{\liminf _{n \rightarrow \infty} \frac{f\left(t_{n} d_{n}\right)}{t_{n}} \mid t_{n} \rightarrow+\infty, d_{n} \rightarrow d\right\},
$$

where $\left\{t_{n}\right\}$ and $\left\{d_{n}\right\}$ are sequences in $\mathbb{R}$ and $X$, respectively.

As shown in [1, Corollary 2.5.1], $\left(I_{\Omega}\right)_{\infty}=I_{\Omega_{\infty}}$ where $I_{\Omega}$ is the indicator function to $\Omega$, i.e.,

$$
I_{\Omega}(x)= \begin{cases}0 & \text { if } x \in \Omega \\ +\infty & \text { otherwise }\end{cases}
$$

Lemma 2.1. ([1, Proposition 2.6.1]). Let $f_{i}: X \rightarrow \mathbb{R} \cup\{+\infty\}, i=1, \ldots, p$, be a collection of proper functions, $f:=\sum_{i=1}^{p} f_{i}$, and suppose that $f$ is proper, i.e., $\operatorname{dom} f=\cap_{i=1}^{p} \operatorname{dom} f_{i} \neq \emptyset$. Then $f_{\infty}(d) \geq \sum_{i=1}^{p}\left(f_{i}\right)_{\infty}(d)$ for all d satisfying the condition: if $\left(f_{i}\right)_{\infty}(d)=+\infty$ (respectively $\left.-\infty\right)$ for some $i$, then $\left(f_{j}\right)_{\infty}(d)>-\infty$ (respectively $<+\infty$ ) for $j \neq i$.

If in addition all the functions are l.s.c. and convex, then the equality holds in the inequality.

The function $f: X \rightarrow \mathbb{R} \cup\{+\infty\}$ is said to be coercive if $f_{\infty}(d)>0$ for all $d \neq 0$. The following result is from [1, Propositions 3.1.2 and 3.1.3].

Lemma 2.2. Let $f: X \rightarrow \mathbb{R} \cup\{+\infty\}$ be proper, l.s.c. and convex, then the following three statements are equivalent:

(i) $f$ is coercive;

(ii) the optimal set $\{x \in X \mid f(x)=\inf f\}$ is nonempty and compact;

(iii) $\liminf _{\|x\| \rightarrow+\infty} \frac{f(x)}{\|x\|}>0$.

As an immediate consequence of [1, Theorem 3.4.1], we have the following result.

Lemma 2.3. Let $f: X \rightarrow \mathbb{R} \cup\{+\infty\}$ be l.s.c. and proper. If the optimal set

$$
\{x \in X \mid f(x)=\inf f\} \neq \emptyset,
$$

then $f_{\infty}(d) \geq 0$ for all $d \neq 0$. 


\section{Viscosity-type ApProximation Algorithm}

In this section we develop a new viscosity-type approximation algorithm for finding efficient solutions to the vector optimization problem (1.3) based on using the so-called coercive viscosity vector functions. For the scalar case, the reader can find more information and references on this remarkable class of functions and their applications to optimization in [2].

By a coercive viscosity vector function (see [8] for the more strict type) $G: X \rightarrow$ $C \cup\left\{+\infty_{C}\right\}$ we mean a proper, $C$-convex, positively lower semicontinuous vector function with $\operatorname{dom} G$ a closed convex set. Moreover, this function is continuous and Gateaux differentiable on $\operatorname{dom} G$ and its Gateaux derivative $D G(\cdot)$ enjoys the following properties:

(a) $D G(\cdot)$ is continuous on $\operatorname{dom} G$;

(b) there exists $x^{\prime} \in \operatorname{dom} G$ such that $D G\left(x^{\prime}\right)=0$.

The mapping $G: \mathbb{R}^{k} \rightarrow \mathbb{R}_{+}^{k}$ defined by

$$
G(x)=\|x\|^{2} e_{1}+\|x\|^{2} e_{2}+\cdots+\|x\|^{2} e_{k}
$$

where $e_{1}=(1,0, \cdots, 0), e_{2}=(0,1, \cdots, 0), \cdots, e_{k}=(0,0, \cdots, 1) \in \mathbb{R}^{k}$ is a simple example for a coercive viscosity vector function.

Let us now describe the scheme of the viscosity-type approximation algorithm for finding efficient solutions to (1.3) developed in this section and called for simplicity VTA. The new algorithm does not impose any interiority assumptions on the ordering cone under consideration while using the standing assumptions formulated in Section 2 with some $r \in(0,1]$ from (2.6) as well as use $r$-proper efficient solutions to the subproblems instead of weakly efficient ones as in [8].

VTA requires the following data: a sequence of positive real numbers $\left\{\alpha_{n}\right\}$ and a sequence $\left\{\beta_{n}\right\} \subset[0,1]$ satisfying $\alpha_{n} \rightarrow 0, \beta_{n} \rightarrow 0$. VTA generates a sequence of iterates $\left\{x_{n}\right\} \subset X$ in the following way:

Initialization: Choose $x_{0} \in \operatorname{dom} F$.

Stopping rule: If $x_{n} \in \operatorname{Argmin}_{C}\{F(x) \mid x \in X\}$ for the iterate $x_{n}$, then we let $x_{n+p}=x_{n}$ for all $p \geq 1$.

Iterative step: If $x_{n} \notin \operatorname{Argmin}_{C}\{F(x) \mid x \in X\}$ for the iterate $x_{n}$, we first compute $z_{n}$ by

$$
z_{n} \in \operatorname{Argmin}_{C}^{r p}\left\{F(x)+\alpha_{n} G(x) \mid x \in \Omega_{n}\right\}
$$

with the set $\Omega_{n}=\left\{x \in X \mid F(x) \preceq_{C} F\left(x_{n}\right)\right\}$ and $r \in(0,1]$ from (2.6) and then take as the next iterate any $x_{n+1} \in X$ satisfying

$$
x_{n+1}=\left(1-\beta_{n}\right) z_{n}+\beta_{n} x_{n} .
$$


To justify the well-posedness of VTA and show that either the sequence generated by the method converges to an efficient solution or its cluster points belong to the set of all efficient solutions of (1.3) in the next theorem, we keep the standing assumptions of the paper formulated in Section 2 and impose the following assumptions:

(A) The set $\operatorname{Argmin}_{C}{ }^{r p}\{F(x) \mid x \in X\}$ is nonempty and bounded.

(B) (see [7]) $F$ is $C^{+}$-asymptotically uniformly continuous, that is, for every bounded sequences $\left\{x_{n}\right\},\left\{z_{n}\right\} \subset X$ such that $\left\|x_{n}-z_{n}\right\| \rightarrow 0$ as $n \rightarrow \infty$ and each sequence $\left\{\hbar_{n}\right\} \subset C^{+}$converging to some $\hbar \in C^{+}$, it holds:

$$
\left\langle F\left(x_{n}\right)-F\left(z_{n}\right), \hbar_{n}-\hbar\right\rangle \rightarrow 0 \text { as } n \rightarrow \infty .
$$

Theorem 3.4. In addition to the standing assumptions on the data of problem (1.3) formulated in Section 2, suppose that the assumptions (A) and (B) are fulfilled. Then we have:

(i) VTA is well defined, i.e., the sequence of iterates $\left\{x_{n}\right\}$ is determined for any starting point $x_{0} \in \operatorname{dom} F$ from the initiation step.

(ii) Either $\left\{x_{n}\right\}$ converges to an efficient solution of (1.3) or every cluster point of $\left\{x_{n}\right\}$ in VTA belongs to the set of all efficient solutions of (1.3).

Proof. We devide the proof of the theorem into three steps.

Step 1: Justification of well-posedness of the method. Choose $x_{0} \in \operatorname{dom} F$ in the initiation step and assume that the algorithm reaches the $n$th iteration $x_{n}$, $n=0,1, \ldots$. Let us show that the next iteration can be constructed according to the algorithm scheme, i.e., $x_{n+1}$ exists. By the stopping rule of the algorithm this is certainly the case if $x_{n} \in \operatorname{Argmin}_{C}\{F(x) \mid x \in X\}$.

Otherwise, i.e., when condition $x_{n} \notin \operatorname{Argmin}_{C}\{F(x) \mid x \in X\}$ is satisfied for the fixed $n=0,1, \ldots$ By the assumption (A) and (2.5), there exist $\hbar \in K_{r} \backslash\{0\}$ and $\hat{x} \in \operatorname{Argmin}_{C}{ }^{r p}\{F(x) \mid x \in X\}$ such that

$$
\hat{x} \in \operatorname{argmin}\{\langle F(x), \hbar\rangle \mid x \in X\} .
$$

By the $C$-convexity and positively lower semicontinuity of $F$, we have $\langle F(\cdot), \hbar\rangle$ is convex and 1.s.c. on $X$. This implies that $\operatorname{argmin}\{\langle F(x), \hbar\rangle \mid x \in X\}$ is closed and thus compact by using again the assumption (A) and the fact that

$$
\operatorname{argmin}\{\langle F(x), \hbar\rangle \mid x \in X\} \subset \operatorname{Argmin}_{C}^{r p}\{F(x) \mid x \in X\} .
$$

By virtue of Lemma 2.2, we have

$$
(\hbar F)_{\infty}(d)>0 \quad \forall d \neq 0,
$$


where $(\hbar F)(x)=\langle F(x), \hbar\rangle$ for all $x \in X$. Define further a function $\varphi_{n}: X \rightarrow$ $\mathbb{R} \cup\{+\infty\}$ by

$$
\varphi_{n}(x):=\langle F(x), \hbar\rangle+I_{\Omega_{n}}(x)+\alpha_{n}\langle G(x), \hbar\rangle,
$$

where $\Omega_{n}:=\left\{x \in X \mid F(x) \preceq_{C} F\left(x_{n}\right)\right\}$. By the $C$-convexity and positively lower semicontinuity of $F$ and $G$ we conclude that the scalarized functions $\langle F(\cdot), \hbar\rangle$ and $\langle G(\cdot), \hbar\rangle$ are convex and 1.s.c. as well as $\Omega_{n}$ is convex and closed, and thus the extended function $\varphi$ is 1.s.c. and convex. From the definition of the indicator function, we have

$$
\left(I_{\Omega_{n}}\right)_{\infty}(d)=I_{\left(\Omega_{n}\right)_{\infty}}(d)= \begin{cases}0 & \text { if } d \in\left(\Omega_{n}\right)_{\infty} \\ +\infty & \text { otherwise. }\end{cases}
$$

Besides, from the property (b) of $G$, there exists $x^{\prime} \in \operatorname{dom} G$ such that

$$
\left\langle D G\left(x^{\prime}\right), \hbar\right\rangle=0,
$$

which implies that the following optimization problem:

$$
\min \{\langle G(x), \hbar\rangle \mid x \in X\}
$$

has a nonempty solution set. According to Lemma 2.3, we deduce that

$$
(\hbar G)_{\infty}(d) \geq 0 \quad \forall d \neq 0 .
$$

Combining (3.3), (3.5) with (3.6) and using Lemma 2.1, we get

$$
\left(\varphi_{n}\right)_{\infty}(d)=(\hbar F)_{\infty}(d)+\left(I_{\Omega_{n}}\right)_{\infty}(d)+(\hbar G)_{\infty}(d)>0 \quad \forall d \neq 0 .
$$

Thus it allows us to establish the existence of minimizers for $\varphi_{n}$ by Lemma 2.2. By (2.5) we observe that such a minimizer satisfies (3.1) and can be taken as $z_{n}$. Hence the next iterate $x_{n+1}$ computed by (3.2) is well-defined.

Step 2: Boundedness of the sequence $\left\{x_{n}\right\}$ and proximity of consecutive iterates. If the stopping rule applies at some iteration, then the sequence of iterates remains constant thereafter; thus it converges to the stopping iterate which is an efficient solution of (1.3). From now on we assume without loss of generality that the stopping rule never applies.

We shall show that there exists $M>0$ such that $\left\|x_{n}\right\| \leq M$ for all $n$. Suppose for contradiction that $\left\|x_{n}\right\| \rightarrow+\infty$ as $n \rightarrow \infty$. By (3.3) and Lemma 2.2, we have

$$
\liminf _{\left\|x_{n}\right\| \rightarrow+\infty} \frac{\left\langle F\left(x_{n}\right), \hbar\right\rangle}{\left\|x_{n}\right\|}>0 .
$$


On the other hand, by the fact that $z_{n} \in \Omega_{n}$ and the $C$-convexity of $F$, we deduce from (3.2) that

$$
F\left(x_{n+1}\right) \preceq_{C}\left(1-\beta_{n}\right) F\left(z_{n}\right)+\beta_{n} F\left(x_{n}\right) \preceq_{C} F\left(x_{n}\right) \quad \forall n .
$$

Thus $F\left(x_{n}\right) \preceq_{C} F\left(x_{0}\right)$ for all $n$ and so $\left\langle F\left(x_{n}\right), \hbar\right\rangle \leq\left\langle F\left(x_{0}\right), \hbar\right\rangle$ for all $n$. This implies that

$$
\liminf _{\left\|x_{n}\right\| \rightarrow+\infty} \frac{\left\langle F\left(x_{n}\right), \hbar\right\rangle}{\left\|x_{n}\right\|} \leq \liminf _{\left\|x_{n}\right\| \rightarrow+\infty} \frac{\left\langle F\left(x_{0}\right), \hbar\right\rangle}{\left\|x_{n}\right\|}=0 .
$$

Combining (3.7) with (3.9) gives a contradiction. Thus the sequence $\left\{x_{n}\right\}$ must be bounded. A similar argument shows that $\left\{z_{n}\right\}$ is also bounded.

Step 3: Optimality of the cluster points of the sequence $\left\{x_{n}\right\}$. Since $\left\{x_{n}\right\}$ is bounded, it has some cluster points. We shall show that all of cluster points are efficient solutions of (1.3). Let $\bar{x}$ be one of the cluster points of $\left\{x_{n}\right\}$ and $\left\{x_{n_{k}}\right\}$ be a subsequence of $\left\{x_{n}\right\}$ which converges to $\bar{x}$. To justify next that $\bar{x}$ is an efficient solution to the vector optimization problem (1.3), consider the scalarized function $\psi_{\hbar}(x)=\langle F(x), \hbar\rangle$ and show that

$$
\psi_{\hbar}(\bar{x}) \leq \psi_{\hbar}\left(x_{n}\right) \text { for all } \hbar \in C^{+} \text {and } n \geq 0,
$$

where the positive polar $C^{+}$is defined in (2.1). Since $F$ is assumed to be positively lower semicontinuous and $C$-convex, the function $\psi_{\hbar}: X \rightarrow \mathbb{R} \cup\{+\infty\}$ is lower semicontinuous and convex and so $\psi_{\hbar}(\bar{x}) \leq \liminf _{k \rightarrow \infty} \psi_{\hbar}\left(x_{n_{k}}\right)$. Besides by (3.8), we have $F\left(x_{n+1}\right) \preceq_{C} F\left(x_{n}\right)$. Thus

$$
\psi_{\hbar}\left(x_{n+1}\right) \leq \psi_{\hbar}\left(x_{n}\right) \quad \forall n \geq 0 .
$$

Consequently, $\liminf _{k \rightarrow \infty} \psi_{\hbar}\left(x_{n_{k}}\right)=\inf \left\{\psi_{\hbar}\left(x_{n}\right)\right\}$ which gives $\psi_{\hbar}(\bar{x}) \leq \inf \left\{\psi_{\hbar}\left(x_{n}\right)\right\}$. Hence we have (3.10) and conclude from it that

$$
F(\bar{x}) \preceq_{C} F\left(x_{n}\right) \text { for all } n \geq 0 .
$$

Since $z_{n}$ solves the auxiliary vector optimization problem in (3.1), we get by (2.5) that there is $\hbar_{n} \in K_{r} \backslash\{0\}$ with some $r \in(0,1]$ from (2.6) such that $z_{n}$ also solves the problem

$$
\min \eta_{n}(x) \text { subject to } x \in \Omega_{n},
$$

where $\eta_{n}: X \rightarrow \mathbb{R} \cup\{+\infty\}$ is defined by

$$
\eta_{n}(x):=\left\langle F(x), \hbar_{n}\right\rangle+\alpha_{n}\left\langle G(x), \hbar_{n}\right\rangle .
$$


Since the solution to (3.13) is not altered through multiplication of $\hbar_{n}$ by positive scalars, we assume without loss of generality that $\left\|\hbar_{n}\right\|=1$ for all $n \geq 0$. It follows from the definitions of $\Omega_{n}$ and $G$ that $\Omega_{n} \subset \operatorname{dom} F$ and so $\Omega_{n} \cap \operatorname{dom} G$ is a closed convex set and is contained in $\operatorname{dom} F$. As $F$ is positively partially continuous and $G$ is continuous on $\operatorname{dom} G$, we have $\eta_{n}$ is continuous on $\Omega_{n} \cap \operatorname{dom} G$. Since $z_{n}$ satisfies the first order optimality conditions for the convex problem (3.13), applying now [18, Theorem 3.16], we find $u_{n} \in X$ such that

$$
u_{n} \in \partial \eta_{n}\left(z_{n}\right) \text { and }\left\langle u_{n}, x-z_{n}\right\rangle \geq 0
$$

for all $x \in \Omega_{n}$. We conclude from (3.14) and (3.15) that

$$
u_{n}=v_{n}+\alpha_{n}\left\langle D G\left(z_{n}\right), \hbar_{n}\right\rangle \text { with some } v_{n} \in \partial \psi_{\hbar_{n}}\left(z_{n}\right) .
$$

Assume on the contrary that $\bar{x}$ is not efficient for (1.3). Then there exists $\tilde{x} \in X$ such that

$$
F(\tilde{x}) \preceq_{C} F(\bar{x}) \text { and } F(\tilde{x}) \neq F(\bar{x}) .
$$

Recall that $\hbar_{n} \in K_{r} \backslash\{0\}$ for the restricted polar (2.4) with some $r \in(0,1]$ from (2.6) and that $\left\|\hbar_{n}\right\|=1$ for all $n \geq 0$. So there is a subsequence $\left\{\hbar_{n_{k}}\right\}$ of $\left\{\hbar_{n}\right\}$ which converges to some point $\bar{\hbar} \in Y$ with $\|\bar{\hbar}\|=1$. Let us show that $\bar{\hbar} \in C_{s}^{+}$ where the strict polar $C_{s}^{+}$is defined in (2.2). Indeed, for every $y \in C$ we get $\left\langle y, \hbar_{n_{k}}\right\rangle \geq r\|y\|$. Since the linear form $\hbar \mapsto\langle y, \hbar\rangle$ is continuous, by passing to the limit as $k \rightarrow \infty$, we arrive at $\langle y, \bar{\hbar}\rangle \geq r\|y\|$. The latter ensures that $\bar{\hbar} \in C_{s}^{+}$. Observe that the established assertion $\bar{\hbar} \in C_{s}^{+}$implies together with (3.17) the strict inequality

$$
\langle F(\bar{x})-F(\tilde{x}), \bar{\hbar}\rangle>0 .
$$

On the other hand, from (3.2) and the convexity of $\psi_{\hbar_{n_{k}}}$ we get

$$
\begin{aligned}
& \left\langle F\left(x_{n_{k}+1}\right), \hbar_{n_{k}}\right\rangle \\
= & \psi_{\hbar_{n_{k}}}\left(x_{n_{k}+1}\right)=\psi_{\hbar_{n_{k}}}\left(\beta_{n_{k}} x_{n_{k}}+\left(1-\beta_{n_{k}}\right) z_{n_{k}}\right) \\
\leq & \beta_{n_{k}} \psi_{\hbar_{n_{k}}}\left(x_{n_{k}}\right)+\left(1-\beta_{n_{k}}\right) \psi_{\hbar_{n_{k}}}\left(z_{n_{k}}\right) \\
= & \beta_{n_{k}}\left(\psi_{\hbar_{n_{k}}}\left(x_{n_{k}}\right)-\psi_{\hbar_{n_{k}}}\left(z_{n_{k}}\right)\right)+\psi_{\hbar_{n_{k}}}\left(z_{n_{k}}\right) \\
= & \beta_{n_{k}}\left\langle F\left(x_{n_{k}}\right)-F\left(z_{n_{k}}\right), \hbar_{n_{k}}\right\rangle+\psi_{\hbar_{n_{k}}}\left(z_{n_{k}}\right) \\
= & \beta_{n_{k}}\left\langle F\left(x_{n_{k}}\right)-F\left(z_{n_{k}}\right), \hbar_{n_{k}}-\bar{\hbar}\right\rangle+\beta_{n_{k}}\left(\psi_{\bar{\hbar}}(\bar{x})-\psi_{\bar{\hbar}}\left(z_{n_{k}}\right)\right) \\
& +\beta_{n_{k}}\left(\psi_{\bar{\hbar}}\left(x_{n_{k}}\right)-\psi_{\bar{\hbar}}(\bar{x})\right)+\psi_{\hbar_{n_{k}}}\left(z_{n_{k}}\right) .
\end{aligned}
$$


Furthermore, it follows from (3.12) and (3.17) that $F(\tilde{x}) \preceq_{C} F(\bar{x}) \preceq_{C} F\left(x_{n}\right)$ and hence $\tilde{x} \in \Omega_{n}$ for all $n \geq 0$. The latter implies together with (3.15) and (3.16) that

$$
\begin{aligned}
& \psi_{\hbar_{n_{k}}}(\tilde{x})-\psi_{\hbar_{n_{k}}}\left(z_{n_{k}}\right) \\
\geq & \left\langle v_{n_{k}}, \tilde{x}-z_{n_{k}}\right\rangle \\
= & \left\langle u_{n_{k}}, \tilde{x}-z_{n_{k}}\right\rangle-\alpha_{n_{k}}\left\langle D G\left(z_{n_{k}}\right), \hbar_{n_{k}}\right\rangle\left(\tilde{x}-z_{n_{k}}\right) \\
\geq & -\alpha_{n_{k}}\left\langle D G\left(z_{n_{k}}\right), \hbar_{n_{k}}\right\rangle\left(\tilde{x}-z_{n_{k}}\right) \\
\geq & -\alpha_{n_{k}}\left\|D G\left(z_{n_{k}}\right)\right\| \cdot\left\|\tilde{x}-z_{n_{k}}\right\| .
\end{aligned}
$$

Using now (3.10), we derive from (3.19) and (3.20) that

$$
\begin{aligned}
& \left\langle F(\tilde{x})-F(\bar{x}), \hbar_{n_{k}}\right\rangle \\
\geq & \left\langle F(\tilde{x})-F\left(x_{n_{k}+1}\right), \hbar_{n_{k}}\right\rangle=\psi_{\hbar_{n_{k}}}(\tilde{x})-\psi_{\hbar_{n_{k}}}\left(x_{n_{k}+1}\right) \\
\geq & \psi_{\hbar_{n_{k}}}(\tilde{x})-\psi_{\hbar_{n_{k}}}\left(z_{n_{k}}\right)-\beta_{n_{k}}\left(\left\langle F\left(x_{n_{k}}\right)-F\left(z_{n_{k}}\right), \hbar_{n_{k}}-\bar{\hbar}\right\rangle\right. \\
& -\beta_{n_{k}}\left(\psi_{\bar{\hbar}}(\bar{x})-\psi_{\bar{\hbar}}\left(z_{n_{k}}\right)\right)-\beta_{n_{k}}\left(\psi_{\bar{\hbar}}\left(x_{n_{k}}\right)-\psi_{\bar{\hbar}}(\bar{x})\right) \\
\geq & -\alpha_{n_{k}}|| D G\left(z_{n_{k}}\right)|| \cdot|| \tilde{x}-z_{n_{k}}|| \\
& -\beta_{n_{k}}\left(\left\langle F\left(x_{n_{k}}\right)-F\left(z_{n_{k}}\right), \hbar_{n_{k}}-\bar{\hbar}\right\rangle-\beta_{n_{k}}\left(\psi_{\bar{\hbar}}(\bar{x})-\psi_{\bar{\hbar}}\left(z_{n_{k}}\right)\right)\right. \\
& -\beta_{n_{k}}\left(\psi_{\bar{\hbar}}\left(x_{n_{k}}\right)-\psi_{\bar{\hbar}}(\bar{x})\right) .
\end{aligned}
$$

We next take the inferior limit of the rightmost expression in (3.21). Note that $\lim _{n \rightarrow \infty} \alpha_{n}=0$ and $\left\{\left\|\tilde{x}-z_{n}\right\|\right\}$ is bounded. As $D G(\cdot)$ is continuous, $Y$ is finite dimensional and $\left\{z_{n}\right\}$ is bounded, we conclude that $\left\{D G\left(z_{n}\right)\right\}$ is also bounded. Note further that $\lim _{n \rightarrow \infty} \beta_{n}=0$ and the boundedness of $\left\{x_{n}\right\}$ and $\left\{z_{n}\right\}$, which follows from (3.2) that $\lim _{n \rightarrow \infty}\left\|x_{n+1}-z_{n}\right\|=\lim _{n \rightarrow \infty} \beta_{n}\left\|x_{n}-z_{n}\right\|=0$. This together with the fact that $\lim _{k \rightarrow \infty} x_{n_{k}}=\bar{x}$ imply that $\lim _{k \rightarrow \infty} z_{n_{k}}=\bar{x}$ and therefore $\lim _{k \rightarrow \infty}\left\|x_{n_{k}}-z_{n_{k}}\right\|=0$. The latter together with the assumption (B) ensure that

$$
\lim _{k \rightarrow \infty}\left\langle F\left(x_{n_{k}}\right)-F\left(z_{n_{k}}\right), \hbar_{n_{k}}-\bar{\hbar}\right\rangle=0 .
$$

Meantime, the lower semicontinuity of the function $\psi_{\bar{\hbar}}$ gives us

$$
\liminf _{k \rightarrow \infty} \psi_{\bar{\hbar}}\left(x_{n_{k}}\right) \geq \psi_{\bar{\hbar}}(\bar{x}) .
$$

Thus $\psi_{\bar{\hbar}}(\bar{x}) \leq \liminf _{k \rightarrow \infty} \psi_{\bar{\hbar}}\left(x_{n_{k}}\right) \leq \psi_{\bar{\hbar}}\left(x_{0}\right)$ by (3.11). Since $\lim _{k \rightarrow \infty} \beta_{n_{k}}=0$, we therefore get

$$
\liminf _{k \rightarrow \infty} \beta_{n_{k}}\left(\psi_{\bar{\hbar}}\left(x_{n_{k}}\right)-\psi_{\bar{\hbar}}(\bar{x})\right)=\liminf _{k \rightarrow \infty} \beta_{n_{k}}\left(\liminf _{k \rightarrow \infty} \psi_{\bar{\hbar}}\left(x_{n_{k}}\right)-\psi_{\bar{\hbar}}(\bar{x})\right)=0 .
$$


A similar manner shows that $\liminf _{k \rightarrow \infty} \beta_{n_{k}}\left(\psi_{\bar{\hbar}}\left(z_{n_{k}}\right)-\psi_{\bar{\hbar}}(\bar{x})\right)=0$. By above arguments, we can conclude that the inferior limit of the rightmost expression in (3.21) as $k \rightarrow \infty$ is zero. Hence we get from (3.21) that

$$
\langle F(\tilde{x})-F(\bar{x}), \bar{\hbar}\rangle \geq 0 .
$$

This contradicts (3.18) and thus justifies that $\bar{x}$ is indeed an efficient solution of the vector optimization problem (1.3).

\section{REFERENCES}

1. A. Auslander and M. Teboulle, Asymptotic cones and functions in optimization and variational inequalities, Springer-Verlag, 2003.

2. H. Attouch, Viscosity solutions of minimization problems, SIAM J. Optim., 6(3) (1996), 769-806.

3. S. Bolintinéanu, Approximate efficiency and scalar stationarity in unbounded nonsmooth convex vector optimization problems, J. Optim. Theory Appl., 106 (2000), 265-296.

4. J. F. Bonnans and A. Shapiro, Perturbation Analysis of Optimization Problems, Springer, New York, 2000.

5. H. Bonnel, A. N. Iusem and B. F. Svaiter, Proximal methods in vector optimization, SIAM J. Optim., 15(4) (2005), 953-970.

6. L. C. Ceng, B. S. Mordukhovich and J. C. Yao, Hybrid approximate proximal method with auxiliary variational inequality for vector optimization, J. Optim. Theory Appl., to appear.

7. L. C. Ceng and J. C. Yao, Aproximate proximal methods in vector optimization, European J. Oper. Res., 183(1) (2007), 1-19.

8. Z. Chen, K. Zhao and X. Liu, Generalized viscosity approximation methods in multiobjective optimizations, (submitted).

9. T. D. Chuong, B. S. Mordukhovich and J. C. Yao, Hybrid approximate proximal algorithms for efficient solutions in vector optimization, (submitted).

10. T. D. Chuong and J. C. Yao, Steepest descent methods for critical points in vector optimization problems, (submitted).

11. M. Ehrgott, Multicriteria Optimization, Springer, Berlin, 2005.

12. J. Fliege and B. F. Svaiter, Steepest descent methods for multicriteria optimization, Math. Methods Oper. Res., 51 (2000), 479-494.

13. L. M. Grana Drummond and A. N. Iusem, A projected gradient method for vector optimization problems, Comput. Optim. Appl., 28 (2004), 5-30. 
14. L. M. Grana Drummond and A. N. Iusem, A steepest descent method for vector optimization, J. Comput. Appl. Math., 175 (2005), 395-414.

15. L. M. Grana Drummond, N. Maculan and B. F. Svaiter, On the choice of parameters for the weighting method in vector optimization, Math. Program., 111(1-2) Ser. B, (2008), 201-216.

16. J. Jahn, Theory of vector maximization: Various concepts of efficient solutions, in: Multicriteria Decision Making, Advances in MCDM Models, Algorithms, Theory, and Applications, T. Gal, T. J. Stewart and T. Hanne (eds.), Kluwer, Boston, 1999, pp. 2-1-2-32.

17. D. T. Luc, Theory of Vector Optimization, Lecture Notes in Economics and Mathematical Systems, 319, Springer-Verlag, Berlin, 1989.

18. R. R. Phelps, Convex Functions, Monotone Operators and Differentiability, 2nd ed., Lecture Notes in Math. 1364, Springer, Berlin, 1993.

Thai Doan Chuong

Department of Mathematics,

Dong Thap University,

783 Pham Huu Lau Road,

Cao Lanh City, Dong Thap Province,

Vietnam

E-mail: chuongthaidoan@yahoo.com

Jen-Chih Yao

Department of Applied Mathematics,

National Sun Yat-sen University,

Kaohsiung 804,

Taiwan

E-mail:yaojc@math.nsysu.edu.tw 